\title{
Efficiency of Active Flow Control by a Synthetic Jet Around a Hump
}

\author{
P. Pick ${ }^{\mathrm{a}}$, V. Skála ${ }^{\mathrm{b}}$, and M. Matějka ${ }^{\mathrm{c}}$ \\ Faculty of Mechanical Engineering at CTU in Prague, Department of Fluid Mechanics and Thermodynamics 12112, \\ Technická 4, Prague
}

\begin{abstract}
The present contribution summarizes the efficiency of active flow control of the boundary layer of air around the hump. The synthetic jet generator with rectangular output part, i.e. slot, is actuated using a modulated signal. The modulated signal phase shift and its influence on the loss coefficient is investigated. The synthetic jet influences the character of the boundary layer and the size of the mixing area in a positive way. Decrease of the loss coefficient resulting from the change of the mixing size area (i.e. wake) is investigated. A velocity profile and a pressure profile were measured behind the hump by the CTA probe and the Kiel probe, respectively. Loss coefficients were obtained from total pressure measurements, each with a different measurement setup. These coefficients were compared with each other to show the benefits of the synthetic jet active flow control technique.
\end{abstract}

\section{Introduction}

The synthetic jet boundary layer control is an effective method using an unsteady blowing of the fluid flow into the boundary layer and its periodic suction from the close surroundings. Article [1] summarizes the criteria for, and the region of, the existence of the synthetic jet, and [2] summarizes the rules for the design of a generator that should control the boundary layer. An example of the loss decrease of the flow past the cylinder was provided in [3]. In [4] the influence of the actuation consisting of a carrying and a modulation component was evaluated as was the influence of the phase shift of the modulation frequency. This contribution presents a case of carrying frequency phase shift.

\section{Experimental setup}

A hump of a prismatic shape was placed as the body to be flown past on the bottom and the side wall of the aerodynamic tunnel. The tunnel had an open cycle. The closed test section dimension $300 \times 200 \mathrm{~mm}(\mathrm{~W} \times \mathrm{H})$ was used. The working medium in the tunnel was air.

The hump forms a corner shape generating a corner vortex of the secondary flow. In the hump two synthetic jet generator modules were placed. The horizontal module, embedded in the horizontal hump, consisted of 8 active cells and the vertical module of 5 such cells. Each cell was formed by two acoustic transducers mounted against each other, which moved in phase against each other and sucked fluid from the free surroundings into the cavity and then blew it out through a $1 \mathrm{~mm}$ wide slot. The cells in the modules were connected into two parallel branches in order for the neighbouring cells to be actuatable by different phase shifts. Both synthetic jet modules were actuated by the frequency of $330 \mathrm{~Hz}$, which is both the carrying actuation frequency and the resonance cavity frequency of each cell.

Total pressure is measured by the Kiel probe placed in the plane of $545 \mathrm{~mm}$ from the leading edge of the hump. Velocity of the flow, measured by CTA probe, is measured in the plane $405 \mathrm{~mm}$ from the leading edge of the hump. Dimensions measured area by the CTA probe and Kiel probe were $251 \mathrm{~mm} \times 140.4 \mathrm{~mm}$. The starting points of these measurement areas were situated near the left bottom corner of the wind tunnel test section. Measurement area is not symmetrical; it is shifted to bottom wall and the left side wall (from a free stream view). Therefore, the wall effect of the synthetic jet is not visible on the right side of the wind tunnel.

\footnotetext{
a petr.pick@atlas.cz

bvladislav.skala@fs.cvut.cz

c milanmatejka@hotmail.com
} 


\subsection{Modulation frequency}

The modulation frequency was determined according to instructions in [2] as follows: a velocity profile measurement after the hump was carried out, using a CTA-probe, with zero actuation. The measurement plane for each working point was $405 \mathrm{~mm}$ from the leading edge of the hump; as this working point, design velocity recalculated according to MSA into the Reynolds number was taken. Each such working point is represented by the design velocity and the working Reynolds number as is shown in Table 1.

Table 1. Design velocities and the working Reynolds number

\begin{tabular}{|c|c|}
\hline $\mathbf{U}(\mathbf{m} / \mathbf{s})$ & $\mathbf{R e}$ \\
\hline 5 & 123300 \\
\hline 8 & 197300 \\
\hline 11 & 271300 \\
\hline 15 & 370000 \\
\hline 18 & 444000 \\
\hline
\end{tabular}

Reynolds number is defined as follows:

$$
\operatorname{Re}=\mathrm{U} \cdot \mathrm{X} / \mathrm{v}
$$

$\mathrm{U}$ means free stream speed,

$X$ means characteristic dimension (hump length),

$v$ is the kinematic viscosity,

Subsequently, power spectra were calculated from each measured velocity profile. From these spectra the optimal modulation frequency was determined. This frequency was later used for actuation, both with and without a phase shift.

\subsection{Loss coefficient calculation}

The loss coefficient is defined as follows:

$$
\mathrm{d} \xi=\left(\mathrm{p}_{\mathrm{cRef}}-\mathrm{p}_{\mathrm{cKiel}}\right) / \mathrm{p}_{\mathrm{dRef}}
$$

$\mathrm{p}_{\mathrm{cRef}}$ is the reference total pressure in the wind tunnel, measured at the end of the wind tunnel contraction, $\mathrm{p}_{\mathrm{cKiel}}$ is the total pressure measured by Kiel probe, $\mathrm{p}_{\mathrm{dRef}}$ is the reference dynamic pressure at the end of the wind tunnel contraction.
Total coefficient of loss is defined as follows:

$$
\xi_{\mathrm{x}}=\Sigma\left(\mathrm{dS} \cdot \mathrm{d} \xi_{\mathrm{x}}\right) / \mathrm{S}
$$

$\xi_{\mathrm{x}}$ means loss coefficient summed all calculated loss coefficients in every point weighted by dS of every point $\mathrm{dS}$ means area of one point in measuring plane

$\mathrm{d} \xi_{\mathrm{x}}$ means calculated loss coeficient in one point in measuring plane

$\mathrm{S}$ means measured cross section (sum of all dS)

For each point of the measured profile a loss coefficient of such point is calculated whereupon the total loss coefficient value of all the points is calculated with respect to the region of each point. The hump and the measurement planes are shown in figure 1 .

\subsection{Existence of the synthetic jet flow}

In order to be stable the synthetic jet needs to be located in the existence region. As mentioned in [1], the existence of the synthetic jet depends on the fulfilment of several criteria. An analysis of these criteria was carried out there. Using this analysis the synthetic jet generator was designed. Upon its production its characteristics were measured, i.e. the output velocity depending on the input frequency and the output velocity depending on the input electric current). Subsequently the working region of the synthetic jet used in that work was verified. The working region is mentioned in figure 2 . The working region of the designed synthetic jet generator when operating at the carrying resonant frequency of $330 \mathrm{~Hz}$ is in a sufficient distance from the border of the existence of the synthetic jet. As a result, the existence of the synthetic jet is verified. Figure 2 also shows the working points for the above specified flow velocities in the wind tunnel, the carrying resonant frequency of $330 \mathrm{~Hz}$ and marginal modulating frequencies $16 \mathrm{~Hz}$ and $96 \mathrm{~Hz}$.

\section{Experimental results}

\subsection{CTA probe results}

Modulation frequencies for the working Reynolds numbers were determined using the CTA probe. Table 2 shows a list of carrying and modulation frequencies.
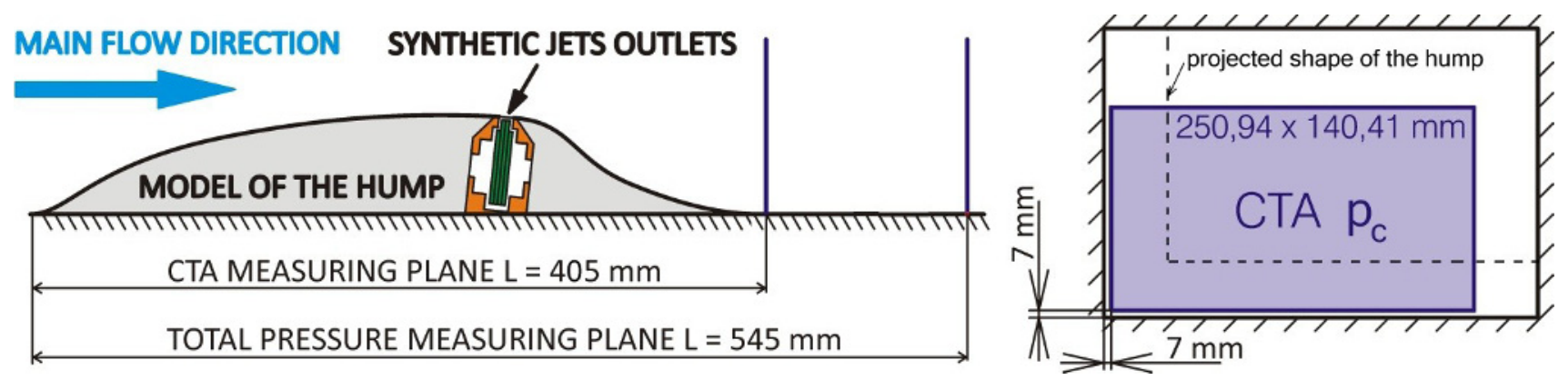

Fig. 1 Model of the hump with synthetic jet (fore-and-aft section), depicting the corner shape of the hump 


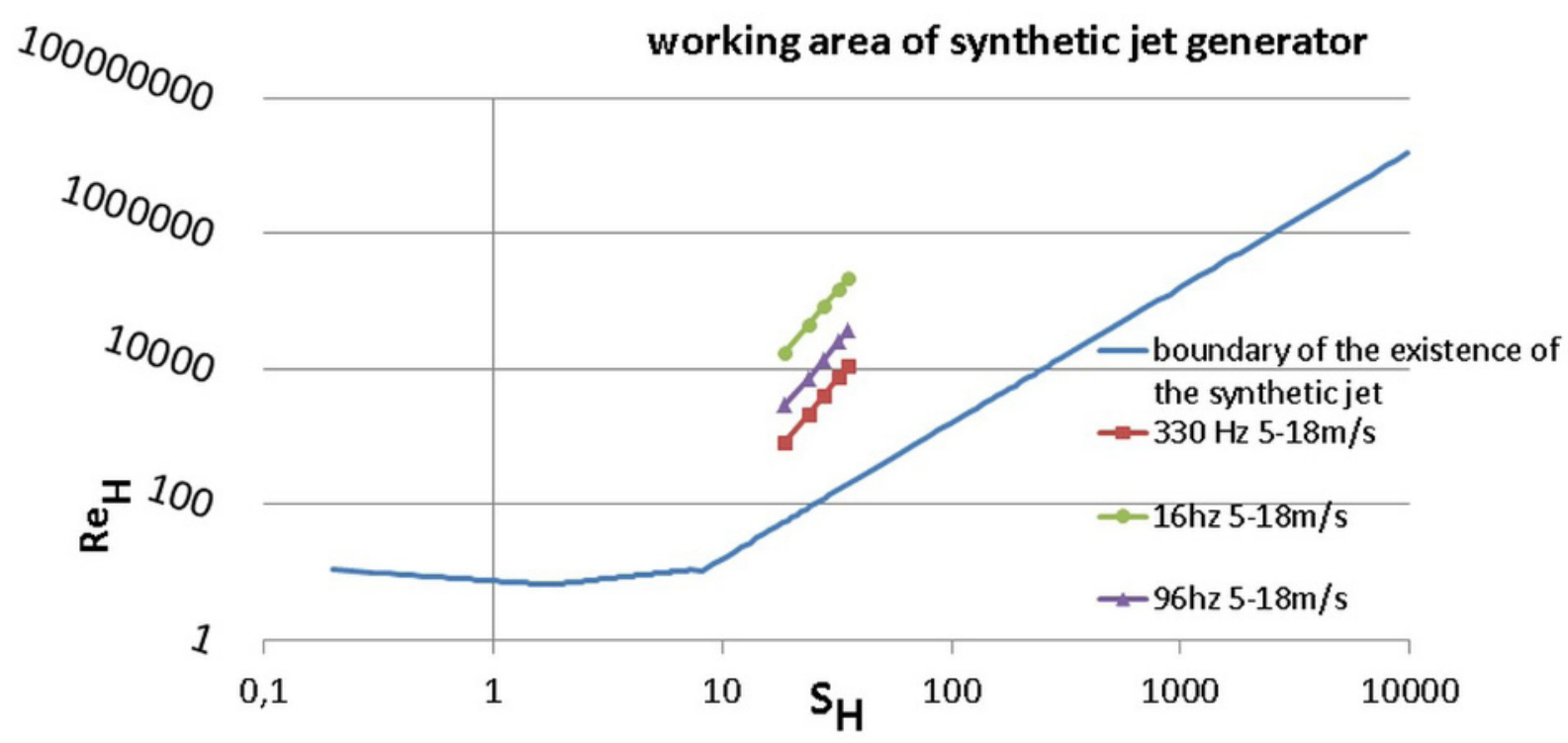

Fig. 2. Working area of the used synthetic jet generators according to design velocities of the measurement

Table 2. Carrying and modulation frequencies for the working Reynolds numbers (Re)

\begin{tabular}{|c|c|c|c|}
\hline $\mathbf{R e}$ & $\mathbf{U} \mathbf{0}(\mathbf{m} / \mathbf{s})$ & $\mathbf{f}_{\mathbf{m}}$ & $\mathbf{f}_{\mathbf{c}}$ \\
\hline 123300 & 7.7 & 16.5 & 330 \\
\hline 197300 & 12.5 & 46 & 330 \\
\hline 271300 & 17.1 & 67.5 & 330 \\
\hline 370000 & 23.5 & 60 & 330 \\
\hline 444000 & 27.6 & 96 & 330 \\
\hline
\end{tabular}

\subsection{Kiel probe results}

A series of pressure profile was obtained by measuring the total pressure. It is depicted on the figure 3 where is provides one of the profiles.

This figure clearly shows the corner vortex arising from secondary flow and also the influence of the nearby wall on the sides of the measurement area.

The total pressure measured by Kiel probe was used for the calculation of the loss coefficient. The loss coefficients are plotted in the graph in figure 4. This picture shows that with an increasing velocity the benefit of actuation on the loss coefficient decrease diminishes.

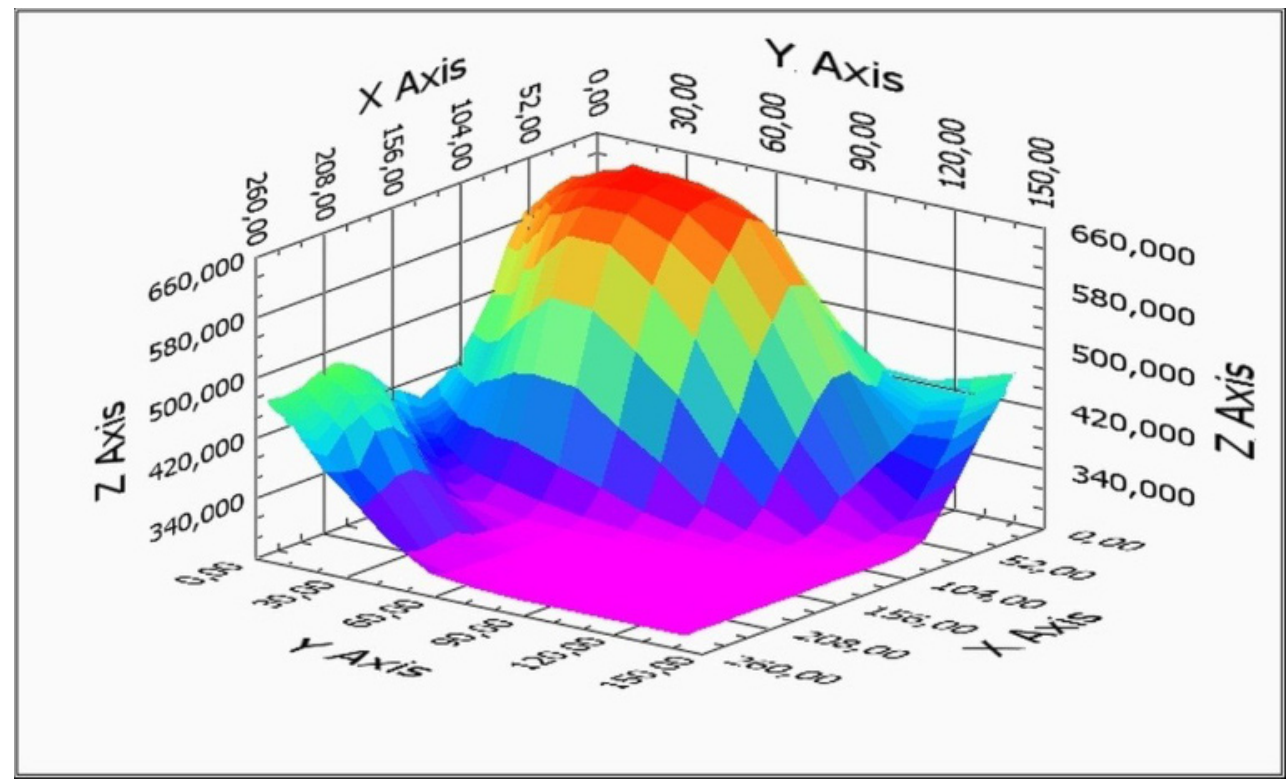

Fig. 3. Total pressure field measured by Kiel probe in the measurement area $545 \mathrm{~mm}$ from the leading edge of the hump, $R e=444000$, no actuation. 


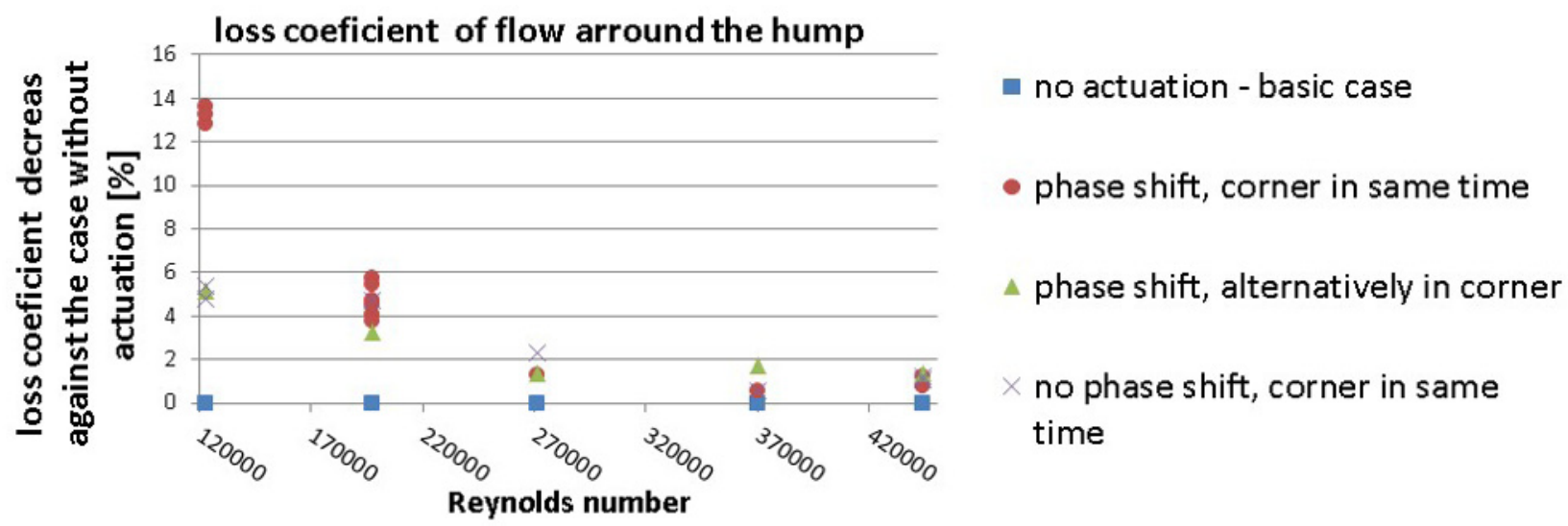

Fig. 4. Loss coefficient decrease against basic case without actuation measured on the working Reynolds numbers

\section{RESULTS}

Figure 4 provides us with interesting information. The greatest loss coefficient decrease was reached with the velocity of approximately $5 \mathrm{~m} / \mathrm{s}(\operatorname{Re} 123300)$; the loss coefficient decreased by $13.2 \%$. In this case the actuation was carried out with a phase shift in carrying frequency and both modules were connected so as to blow against each other simultaneously in the corner when actuated. When the modules were actuated with a phase shift of carrying frequency and connected so as to alternate in blowing, the maximum loss coefficient decrease was reached by $5.1 \%$, with the same velocity of $5 \mathrm{~m} / \mathrm{s}$ ( $\operatorname{Re} 123$ 300). However, in the whole range of velocities the value of the loss coefficient was no lower than $1.3 \%$ in comparison with the base case (without actuation). In case of actuation with zero phase shift on the carrying frequency, the loss coefficient decrease was of similar characteristics but the maximum loss coefficient decrease was $5.3 \%$ with the minimum velocity of $5 \mathrm{~m} / \mathrm{s}$ (Re 123 300). Further performance was similar as in the last case. With the flow velocity of $18 \mathrm{~m} / \mathrm{s}(\operatorname{Re} 444000)$, the loss coefficient decreases to $1.1 \%$ lower value against the case without an actuation.

It was also found that the use of synthesized jet under higher velocities requires a more detailed investigation of the conditions existing in the region of corner vortex and of the secondary flow in order to reach a greater loss coefficient decrease under such velocities. In view of the positive results for the alternate blowing on the corner vortex of the secondary flow it can be predicted for this measurement configuration that the actuation shift in the carrying frequency has the potential to be a more effective type of actuation than shift in the modulation frequency. The measurement data obtained also show the enlargement of the region that is under the influence of the corner vortex of the secondary flow under higher velocities.

\section{CONCLUSION}

On the basis of the experimentally obtained data it was found that the phase shift of the modulation frequency has a positive effect on the decrease of the loss coefficient in the region of the measured velocities. The loss coefficient was decreased by as much as $13.2 \%$. In case of zero phase shift actuation the loss coefficient decrease was reached, the value being $5.3 \%$ at maximum. The most significant influence is gained under lower velocities. Another finding is that a positive influence on the corner vortex of the secondary flow is gained if modules are placed orthogonally against each other and alternate in blowing. As flow velocities increase, the active control decreases in its influence on the boundary layer, which probably occurs due to an unsuitable actuation form for the respective geometry case. The phase shift of the modulation frequency and the alternate blowing of two mutually orthogonal modules can, under the highest velocities, ensure the loss coefficient decrease by $1.3 \%$. Therefore, the positive effect of the modulation frequency phase shift was proved conclusively.

\section{References}

1. Z. Trávníček, Z. Broučková, J. Kordík, AIAA J. 50 (9), 2012-2017 (2012)

2. M. Matejka, P. Doerffer, M. Kurowski, 31. Setkáni kat. mech. tekutin a termomech., 149-152, (2012)

3. M. Matejka, P. Pick, P. Prochazka, J. Nozicka, JFVIP, 2009 (4), 353-365. (2009),

4. P. Pick, M. Andrle, M. Matějka, 31. Setkáni kat. mech. tekutin a termomech., 189-192, (2012) 